\title{
The AGIS metric and time of test: a replication study
}

\author{
Steve Counsell, Stephen Swift, Allan Tucker, \\ Department of Computer Science \\ Brunel University London, United Kingdom \\ steve.counsell@brunel.ac.uk
}

\begin{abstract}
Visual Field (VF) tests and corresponding data are commonly used in clinical practices to manage glaucoma. The standard metric used to measure glaucoma severity is the Advanced Glaucoma Intervention Studies (AGIS) metric. We know that time of day when VF tests are applied can influence a patient's AGIS metric value; a previous study showed that this was the case for a data set of 160 patients. In this paper, we replicate that study using data from 2468 patients obtained from Moorfields Eye Hospital. This may provide further evidence and support of this phenomenon in a replication sense. Results did indeed show a tendency for the metric to be lower for early onset patients in the morning; equally, for advanced patients, the effect was less pronounced. We thus found support for the earlier work of Montolio et al. [4] and add to the body of evidence on the AGIS metric.
\end{abstract}

Keywords-Visual Field, AGIS, glaucoma

\section{INTRODUCTION}

Visual field (VF) data is a standard technique used for managing the condition of glaucoma (deterioration of visual field leading to blindness). Analysis of visual field data has been used widely to predicting glaucoma deterioration; a variety of techniques including data classification, clustering [1] and statistical methods [2, 3] have been used as a vehicle for this. At a more simplistic level, we know that the time of day when a test is applied can influence the value of a VF reading. A previous study by Montolio et al [4] showed that the time of day when a VF test was conducted influenced the test results; 160 eyes from 160 patients were tested. In this paper, we replicate that study with data from both eyes from a larger set of 2468 patients, obtained from Moorfields Eye Hospital (London).

\section{BACKGROUND}

Visual field data is collected by means of computerised automated perimetry commonly used in clinical practices for the diagnosis and monitoring of glaucoma. The visual field locations represent a patient's eye sensitivity to light; the most common tests divide the eye into typically 54 and 76 points (including the blind spot) depending on the type of test and particular variant of glaucoma being screened for. In clinical practices, the Advance Glaucoma Intervention Studies (AGIS) is frequently used for assessing glaucoma severity. The AGIS metric [5] was developed to assess test reliability quantitatively and to measure visual field defect severity and is derived from both the number and depth of adjacent depressed test locations in the nasal area, upper hemi-field, and lower hemi-field (see Fig.1). The AGIS metric is scored from 0 to 20 where categorical glaucoma conditions are derived. The glaucoma conditions include 'none', 'mild', 'moderate', 'severe', and 'end stage' (Table 1).

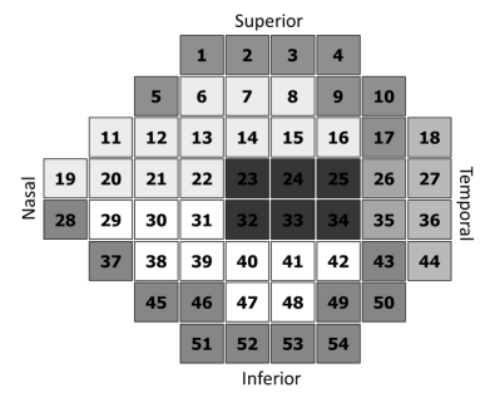

Fig. 1. The fifty-four locations of the VF map

TABLE 1. AGIS CATEGories

\begin{tabular}{|l|l|}
\hline AGIS Score & AGIS Category \\
\hline 0 & None \\
\hline $1-5$ & Mild \\
\hline $6-11$ & Moderate \\
\hline $12-17$ & Severe \\
\hline $18-20$ & End-Stage \\
\hline
\end{tabular}

\section{DATA ANALYSIS}

The data we used consisted of 28778 tests among 2468 patients (using both the left and right eye for each patient). The AGIS value from a number of visits by each patient was collected. The data therefore represents a longitudinal view of data for each patient across a time frame spanning, in most cases, a number of years. Note that we did not consider seconds as part of our analysis (since we considered the granularity that the "hh:mm" format gave us sufficient for, at least, this preliminary study). We then ordered the AGIS scores on time of test. Figure 2 shows the number of tests in each of the hour frames that tests were carried out in the $24 \times 1 \mathrm{hr}$ hour periods (i.e., 00.00-24.00). Figure 2 shows that the majority of tests were undertaken between the hours of 10.00 and 16.00 (in fact, a total of 26676). 


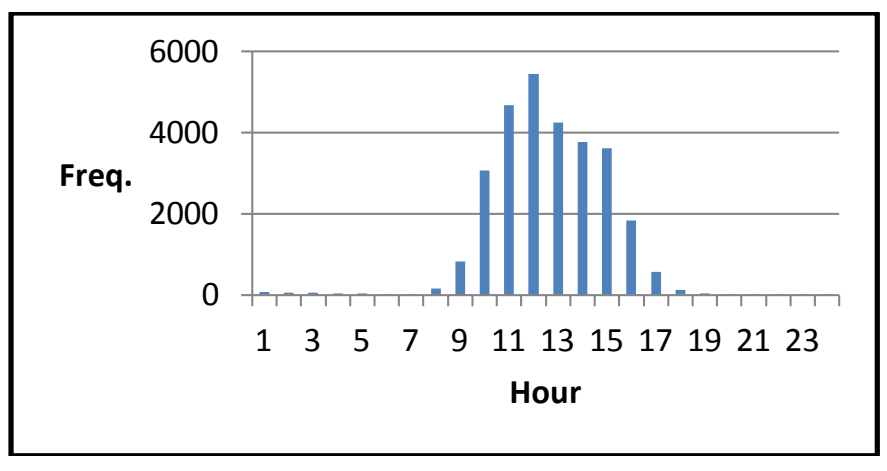

Fig. 2. Frequency of VF tests

The original study by [4] split the day into four periods. These were before 10am, 10am-12, 12-14.00 and after 14.00. According to [4], patients with early glaucoma performed significantly better in the early morning, compared with the rest of the day. In moderate/severe glaucoma, the effect of time of day was less pronounced. Figure 3 shows the mean and median values for the AGIS metric between these four periods of the day: 1) <10am, 2) 10-12, 3) 12-14 and 4) 14-23 for patients with early glaucoma.

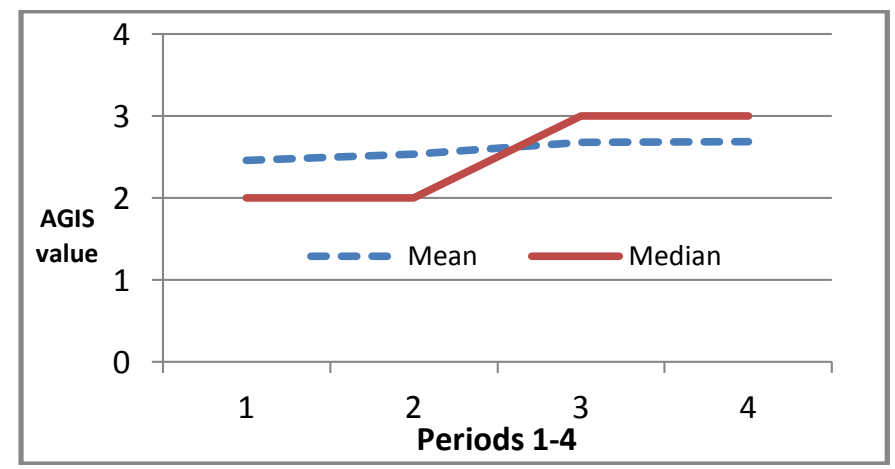

Fig. 3. Early (Mild) glaucoma AGIS results

The values in the figure provide support for the conclusions of the earlier work by [4]. The median rose over the four periods from 2 to 3 (i.e., a $50 \%$ increase). The mean AGIS on the other hand showed less support. Before 10am it was 2.46 and between $10 \mathrm{am}$ and 12 rose only slightly to 2.53 . This compares with 2.68 at both of the other time periods. In terms of numbers in each category, for period 1, there were 1102 observations (i.e., AGIS values in the Mild category); and for periods 2, 3 and 4 there were 2902, 2188 and 1782 values, respectively. Figure 4 shows the AGIS data for the Moderate/Severe category. The data in this figure also supports the earlier work of [4] in which AGIS values across the four periods showed very little variation. The mean values of $10.14,10.17,10.31$ and 10.48 across periods $1-4$, respectively differ by only 0.34 . The median value of AGIS, however, and in contrast to Fig 3, remained static at 10.00 across all periods. The number of AGIS values in each of the four categories was: 1012, 2702, 2826 and 2079, respectively.

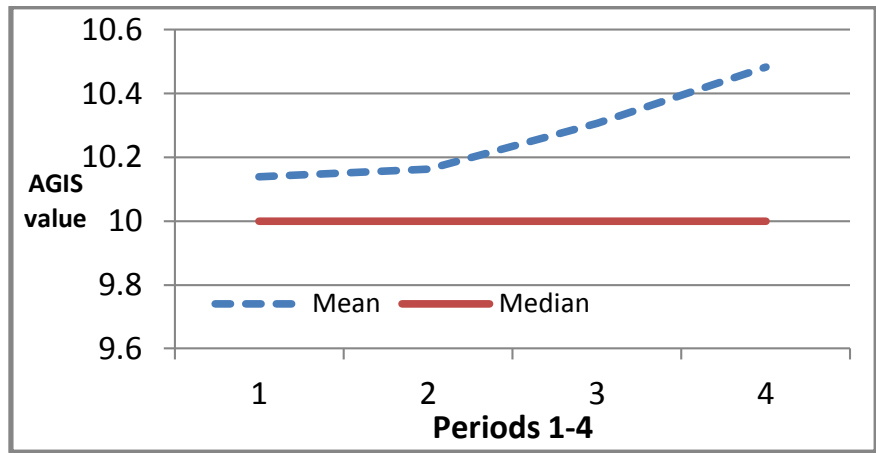

Fig. 4. Moderate/Severe glaucoma AGIS results

We would concur with the results in [4]. In terms of the median values, early glaucoma patients saw a relatively large rise in the AGIS values after 12 midday. For more advanced patients, there was very little change across all four periods.

\section{CONCLUSIONS/FUTURE WORK}

In this paper, we replicated a previous study into the AGIS metric. We found support for the earlier study when contrasting the AGIS metric scores for patients with early and patients with advanced onset of glaucoma. The results add weight to the body of evidence that the time of day of a test does have an influence on the way that results are generated and can be interpreted. Future work will focus on a set of statistical test (not covered in this paper due to space limitations) to determine statistical significance of the results or otherwise. We will also explore each of the 5 categories in Table 1 individually.

\section{ACKNOWLEDGEMENTS}

This work was supported by the Engineering and Physical Sciences Research Council (EPSRC) of the UK, under grant number: EP/H019685/1.

\section{REFERENCES}

[1] S. Ceccon, D. Garway-Heath, D. Crabb and A. Tucker, "Exploring Early Glaucoma and the Visual Field Test: Classification and Clustering Using Bayesian Networks," 2014.

[2] S. Swift and X. Liu, "Predicting glaucomatous visual field deterioration through short multivariate time series modelling," Artif. Intell. Med., vol. 24, pp. 5-24, 2002.

[3] F. W. Fitzke, R. A. Hitchings, D. Poinoosawmy, A. I. McNaught and D. P. Crabb, "Analysis of visual field progression in glaucoma," Br. J. Ophthalmol., vol. 80, pp. 40-48, Jan, 1996.

[4] F. Montolio; C, Wesselink; M, Gordijn; N. Jansonius, "Factors That Influence Standard Automated Perimetry Test Results in Glaucoma: Test Reliability, Technician Experience, Time of Day, and Season". Investigative Ophthalmology \& Visual Science, Vol.53, 7010-7017.

[5] Anonymous "Advanced Glaucoma Intervention Study: 2. Visual Field Test Scoring and Reliability," Ophthalmology, vol. 101, pp. 1445-1455, 8, 1994. 